Volume 3

Number 3 Virtual Platforms: Spaces of Control

and Contestation

2018

\title{
Banerjee, Dholakia and Dholakia, M-Powering Marketing in a Mobile World (2017)
}

Zoila C. Zambrano

University of Texas Rio Grande Valley

Follow this and additional works at: https://digitalcommons.uri.edu/mgdr

Part of the Marketing Commons

\section{Recommended Citation}

Zambrano, Zoila C. (2018) "Banerjee, Dholakia and Dholakia, M-Powering Marketing in a Mobile World (2017)," Markets, Globalization \& Development Review: Vol. 3: No. 3, Article 7.

DOI: 10.23860/MGDR-2018-03-03-07

Available at: https://digitalcommons.uri.edu/mgdr/vol3/iss3/7

This Book Review is brought to you for free and open access by DigitalCommons@URI. It has been accepted for inclusion in Markets, Globalization \& Development Review by an authorized editor of DigitalCommons@URI. For more information, please contact digitalcommons-group@uri.edu. 
Banerjee, Dholakia and Dholakia, M-Powering Marketing in a Mobile World (2017)

\section{Markets, Globalization \& Development Review}
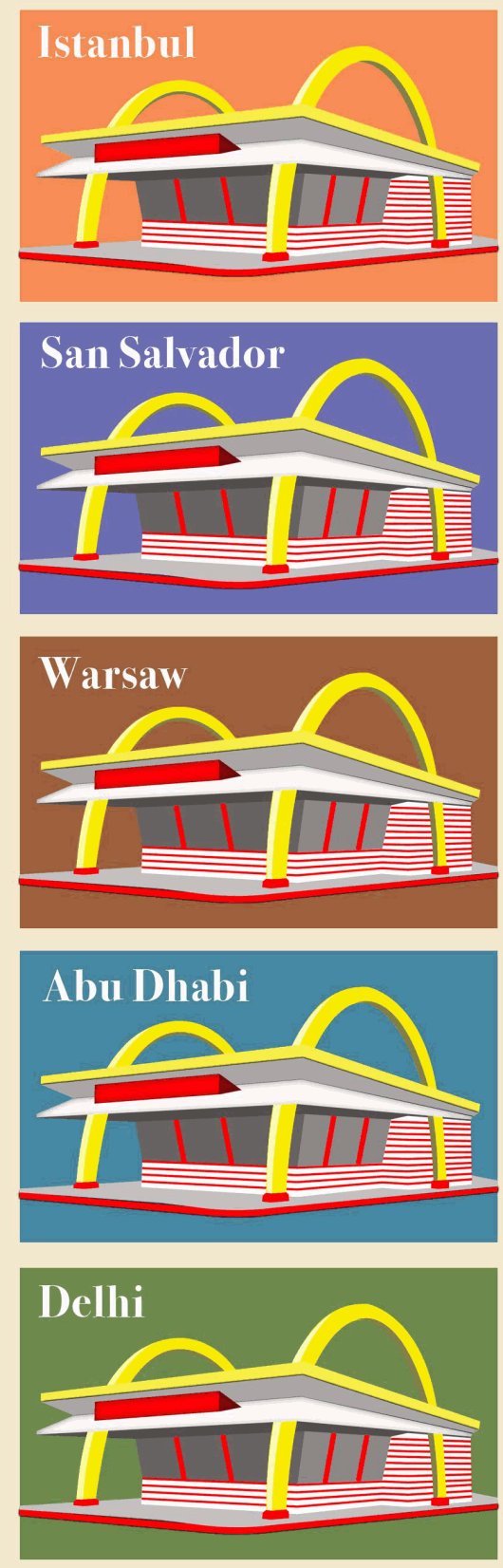
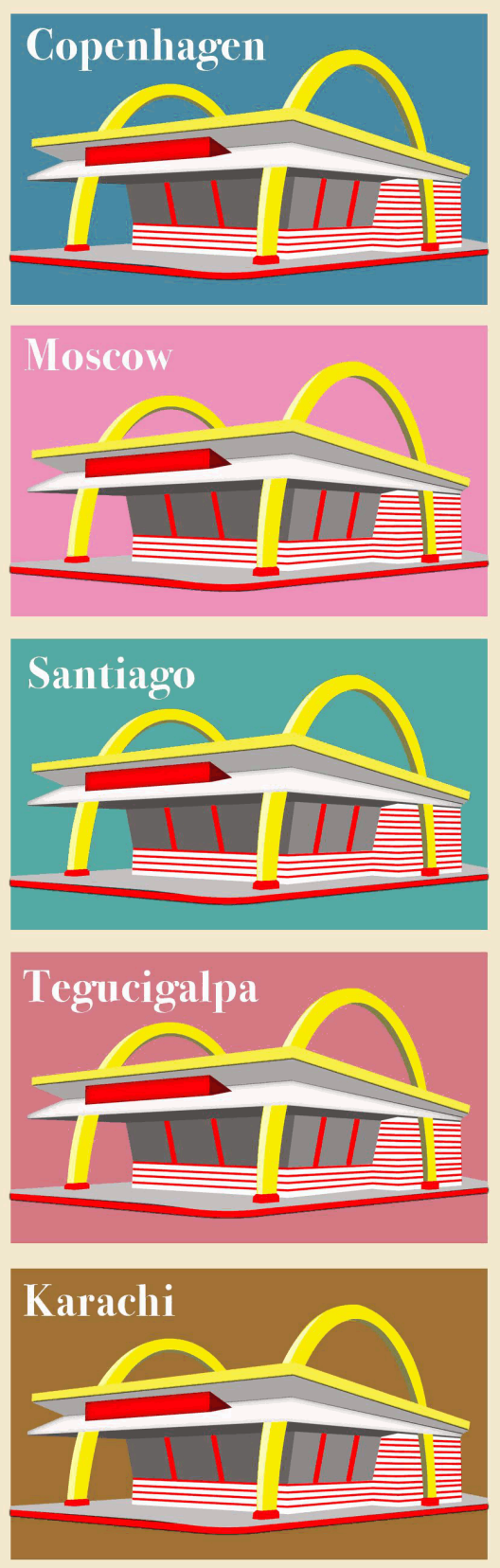
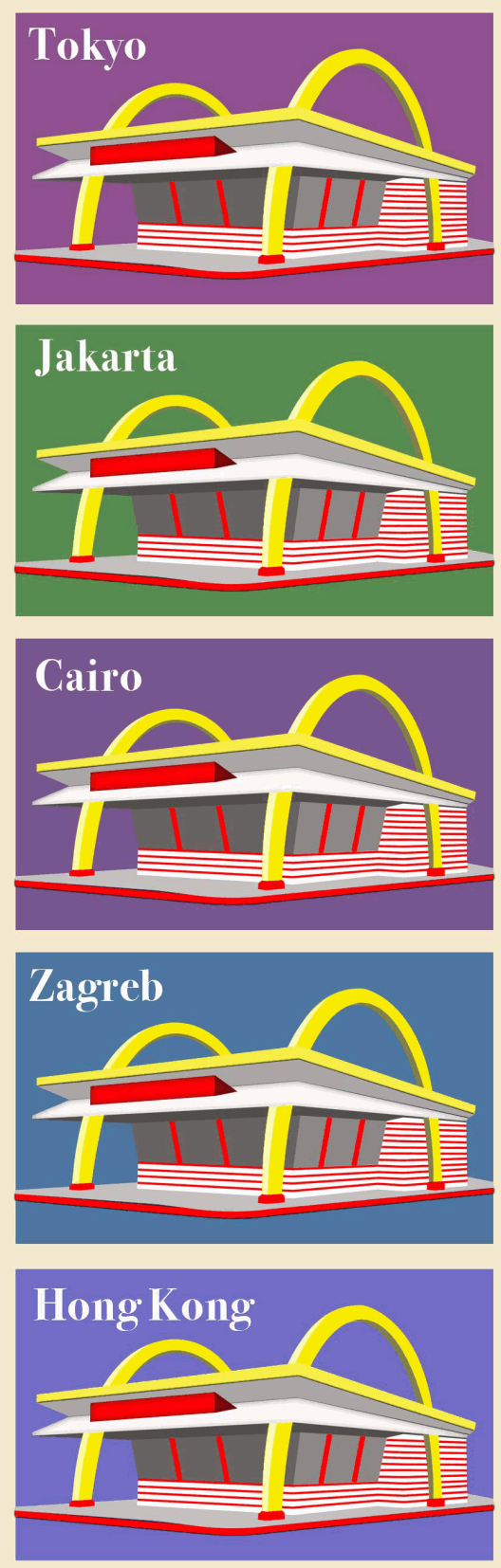

This book review is available in Markets, Globalization \& Development Review: https://digitalcommons.uri.edu/mgdr/ 


\section{Book Review}

\section{Banerjee, Dholakia and Dholakia, M-Powering Marketing in a Mobile World (2017)}

\section{Introduction}

Mobile technology has emerged as the most utilized tool in modern history. Marketers and researchers need to be cognizant of impacts this growth has on the business, marketing, consumer and societal levels. With pervasive ubiquity, worldwide spread of mobile devices and technology has created areas of opportunity for marketing and privacy challenges for global consumers. In the book, M-powering Marketing in a Mobile World, authors Banerjee, Dholakia and Dholakia (2017) provide a broad overview of the complexities associated with rapid global growth of mobile technology and present detailed information along with frameworks to examine opportunities for marketers and convey challenges resulting from mobile growth. Although this book is focused on marketers interested in exploring the benefits of mobile marketing strategies, it succeeds in expanding the understanding of mobile technology and reveals frameworks to assist both readers interested in mobile innovation from a business perspective as well as an academic perspective. In five easy to read, very detailed chapters, the authors provide readers with a practical in-depth introduction to the world of mobile technology innovation and its developments in the global marketplace. The authors begin by introducing readers to the evolution of computer-based internet to mobile voice and data, and implications of the mobile growth on economic, cultural, social and political systems. In the second chapter of the book, various mobile marketing strategies are presented to ensure mobile marketing effectiveness. In the third chapter, readers are insightfully exposed to the roles that mobile applications (apps) play in mobile retail shopping. Chapter four focuses on introducing readers to the multifaceted nature of mobile data and mobile metrics. In the concluding chapter five, the authors discuss at great length the policy implications of privacy and security issues that manifest globally resulting from rapid growth of mobile technology.

\section{A More Detailed Overview of the Chapters}

First, the authors set the stage for the rest of the book by presenting their conceptualization of the mobile revolution and the influences that the rapid growth of mobile is having on various levels of global societies. In their 
mobile revolution framework, ripple effects created by mobile technology affect a country's economic, social, political and cultural structures. Due to the dynamic nature of mobile growth, ripple effects are created by the desire to reach mobile users as well as by the dynamic nature of the mobile phenomena. Both direct and indirect effects of mobile technology impact economic growth. Direct effects are reflected in GDP figures due to the initial investment required to establish mobile network systems along with activities of supply chain firms, software providers, hardware makers and app creators. The authors also delineate the indirect economic effects of mobile growth experienced via highly skilled human capital emergence, increased productivity, increased Foreign Direct Investment (FDI), and increased stock market investments in mobile technology companies. Beyond economic impacts, social effects such as increased connectivity, as well as new eco-systems of partnering banks, developers and financial institutions are also created. Ultimately, with improved communication resulting from the rapid spread of mobile technology, political structures become more open and governmental structures challenged as introductions of democratic ideals and greater individual freedoms are communicated. There is of course also the danger of manipulation of democratic processes, using mobile media (Belk 2017; Dholakia and Reyes 2018). Moreover, mobile multiplier effects influence existing cultural structures directly as well as indirectly. Growth in mobile technology directly influences culture as new entrepreneurial technology modifies the existing culture. Indirect impacts of the mobile revolution on cultures emerge as new groups of entrepreneurs with technological skills enter and change the current cultural capital of nations.

The first chapter also provides a myriad of global examples illustrating the mobile multiplier effects resulting from mobile technology innovation. For instance, none is greater than the purchase of the WhatsApp, a mobile messaging app acquired by Facebook for its mobile global reach of $90 \%$ of Asian, European and Latin American countries. In addition, huge e-commerce growth and emergence of new mobile money systems such as Kenya's m-pesa has changed the market and traditional currency cultures. Also, mobile managed social enterprises have bloomed, such as Blindsquare, a mobile social media company helping the blind maneuver cities via use of sound virtual maps and Ushahidi, an African crowdsourcing platform aiding humanitarian groups. Both microentrepreneurs and established corporations participate in creating mobile innovation solutions such as the small farmer that - out of practical needs - creates a mobile-based system to irrigate his crops remotely; or BMW and Mercedes-Benz, firms that have rolled out car-sharing programs 
made possible by mobile technology. Mobile innovation has changed both affluent economies of Europe, North America and parts of East Asia, as well as developing countries such as those in Africa or Afghanistan.

In the second chapter, the authors present mobile technologies marketers employ to effectively reach marketing goals to obtain customer acquisition or trial, boost engagement, increase sales and satisfaction. These strategies become important as consumers continue to live on their mobile phones and businesses search for opportunities to position their brands through mobile marketing. Changing consumer needs for information has been identified by the authors as the main driver behind the rapid adoption of mobile marketing strategies by businesses primarily due to the ability mobile phones provide to interact effectively with consumers. Mobile strategies must clearly identify desired outcomes so as to differentiate content, to stand out from competitors, to prioritize features of offerings, or synchronize ad creative tactics to reach consumers in real time. The authors introduce a strategy framework which captures the complexities associated with the synchronization of technology platforms, standards, and conventions needed to engage in mobile marketing (Figure 2.8 in the book). The framework presents the combination of online tools (i.e., banners, search, display, native, and applications) and offline tools (i.e., SMS texting) in creating market characteristics which augment reality by enabling the use of QR codes, SMS (texting), and the web. Mobile marketing strategies have become key in the battle between brick-andmortar retailers and online retailers. By adopting mobile marketing strategies, brick-and-mortar retailers have been able to communicate in real time with consumers to encourage them to visit their retail stores, or even a specific department within the store, to take advantage of a mobile marketing promotion. Mobile technology has enabled the development of mobile apps, QR codes and other platforms which capture consumer information and track consumer behaviors - enabling an efficient delivery of marketing mobile messaging as well as the tracking of the customer very closely. The case of Starbucks is presented, which successfully launched a mobile app through which over 4 million dollars in mobile wallet payments are processed weekly and the app continues to engage over 8 million mobile users.

In order for mobile marketing tools to be of value, they must guide in prioritization and planning of mobile strategies. Effective mobile campaigns must clearly identify the goals of the mobile campaign, identifying the product or service being promoted, and identifying the appropriate mobile marketing tool that enables the desired customer engagement. However, despite mobile app creation being limited to larger 
companies, the authors introduce SMS marketing as a tangible strategy smaller mom-and-pop shops may utilize. QR promotional strategy consists of distracting and directing customers to a desired destination whether it be new product information, a survey, to watch a video or sales promotion; all by capturing the QR code on the mobile that redirects the user to the desired destination. Perhaps the most creative use of a QR code mobile marketing strategy the authors presented was employed by Emart, a Korean discount chain. To access promotional coupons, customers were required to scan a shadow $Q R$ code using sunlight limited to times between noon and 1 p.m. Emart employed a type of location-based mobile marketing strategy that companies utilize to reach consumers via push notifications while they were in the shopping process, in a specific part of the store, or within close proximity of the store. Such georeferencing strategies are effective according the authors, as they use push notifications with tailored messages, suggestions, and promotions to engage and motivate consumers to engage with their brand. A case of Elle Style app is presented in which users received mobile strategy promotions resulting in 500,000 visits to stores of affiliated advertisers as well as $12 \%$ retailer engagement.

In the third chapter, the authors focus on mobile retailing and provide details of the sophisticated technologies that make it possible for physical retailers to engage consumers. Faced with a strong fight from online retailers due to a shift in consumer preferences for the convenience and pricing offered online, brick-and-mortar retailers defend the right to retain their consumers by utilizing mobile technology in the form of apps to capture consumer attention to guide customers to their stores. While the mobile users are in their stores, retailers push promotional information to engage customers in shopping. At the same time, customers utilize their mobile devices to access showrooming apps which lure customers away from physical to online retailers, offering better deals and pricing.

The authors present details on the functionality and applicability of technology being used to track consumers' location and movements with the aims of providing consumers with customizable offerings and improved shopping experience. The use of location tracking sensors and supplemental technologies have developed a highly synchronized and inescapable communications system requiring constant information exchange between advertisers, manufacturers, retailers, products and shoppers, commonly referred to as the Internet-of-Things (loT). Hypersurveillance refers to the utilization of highly efficient and accurate smart LED light fixtures to transmit codes to consumers via mobile phones, and cameras to identify exact location of consumers while in a 
retail setting. For example, consumers may be in a certain aisle attempting to decide between two brands of the same product and such hypersurveillance technology allows companies to instantly send a mobile coupon to the consumer for one of the brands.

In the fourth chapter, the authors document the transformation marketing has experienced as a result of growth in mobile data. Not only has this growth created large volumes of data that must be captured but also created the need to mine data to obtain consumer insights to craft effective mobile marketing strategies. Dynamic synchronized data refers the cross-analysis of data from various databases (i.e., mobile phone, temperature, social media, Application Protocol Interface that provide latitude and latitude, Yelp restaurant reviews, etc.), to create a holistic or enhanced mobile marketing offer to the customer at just the correct time. An example could be of a circumstance in which a consumer received the perfect discount on a cold drink, via text message, when they are near a specific restaurant on a very hot day. Such mobile promotion is made possible by pulling data from various databases that capture temperature, humidity, restaurant reviews, the exact location of the consumers and synchronizing that data to deduce that - because of the hot and humid weather - this particular customer may be very interested in having a cold drink at a good nearby restaurant. The authors discuss how the Happy Atmosphere Index ( $\mathrm{HAl}$ ) numbers are extracted from ecosystem, applying analysis software to identify how consumer happiness may be predicted via higher happier ambiance scores.

Mobile marketing partners (MMPs), such as Facebook, assist businesses to effectively spend their social media marketing dollar by connecting businesses to expanded consumer ecosystems. MMPs track, report and analyze integrated data consisting of clicks, conversion rates, installs, impressions, revenues, costs, loyals, ROI (Return on Investment), ARPU (Average Revenue Per User) as well as purchase made within the app. Effective attribution methods are dependent on the ability to successfully track the steps that lead to the specific outcomes, such as clicking on an advertisement or making a purchase. Businesses are able to use various attribution methods to track the outcomes of their mobile advertisements; device fingerprinting, identifier matching and attribution via location tracking. An issue that the authors identify is consumer use of various mobile devices such as mobile phone, tablet, and laptop behaviors that make it difficult to attribute accurately across devices. A solution is presented if a consumer is logged in across all devices using a unique identifier offered by advertisers and publishers. However, only very 
large companies are capable of capturing Big Data data for each customer across various devices.

In the fifth and final chapter, the authors delineate privacy and security issues associated with the rapid growth of mobile communication technology. Growing data associated with mobile technology is captured, analyzed, transmitted and diverted across various data sources revealing some challenging issues with privacy and security worldwide. Specifically, threats originate with weaknesses identified in the layers of the mobile communication ecosystem. These layers consist of operating systems, hardware, apps, subscriptions and networks - all needed to access data required to effectively communicate with consumers. As smart device mobile technology is incorporated into the consumer lifestyles and homes via internet connected devices such as backdoors, baby monitors, and device linked apps, the emerging internet-of-things (IoT) may be exploited by some with the use of malware introduced to tamper with the data. Security and privacy breaches also present a threat to public trust.

National threats to security are manifested through cloud and infrastructure attacks conducted by competitors, individuals or organizations interested in distributing propaganda of fake or deceptive online information (Dholakia and Reyes 2018), fake "likes" endorsements, or ads that sway public opinion. Globally, nations have responded to security and privacy threats differently as some rush to protect citizen information while others rush to protect cultural or political interests. Some countries are beginning to introduce legislation to protect citizens from privacy and security breaches. For example, during Facebook's acquisition of WhatsApp, the European Union (EU) questioned the categorization of WhatsApp as an app rather than a texting telecommunication service. As a result, Facebook agreed to maintain separate datasets of Facebook users and WhatsApp users to appease EU concerns. In addition, in 2017 the EU introduced legislation holding anyone with access to an individual's data responsible during a data breach as well as requiring explicit opt-in requests prior to collecting individual data. In addition, the legislation extended rights to citizens and residents by enabling access to monetary compensation during a data breach - and authority and rights for the consumer to request data deletions or removals. To enforce such legislation, the EU introduced heavy fines for serious violations. On the other hand, it appears that China monitors citizens' electronic activity and blocks access to information perceived as politically or culturally unacceptable by the government via the Great Firewall of China. 
When it comes to securing mobile data in developing countries, the authors share data breach experiences of developing countries and how nations respond to threats. In parts of Latin America, Jumcar malicious code was utilized to compromise customer's banking information via their mobile devices and computers. In addition, the authors present Hong Kong's malware experience when hackers siding with the Chinese authorities flooded anti-China protestor mobile phones with malware. In response to these type of data threats, developing countries launch initiatives to safeguard data. For example, in Colombia, Nequi, a mobile banking service requires face or voice recognition prior to consumer access on mobile devices. Also, Tunisia's e-Dinar - a national electronic currency and e-payment system - applies blockchain cryptographic technology, similar to that of Bitcoin, to safeguard the system. Many examples are presented, one of the most interesting is the use by the Indian government of Aadhaar biometric identification cards linked to approximately 400 million bank accounts, and used to transact over 3 million transactions yearly.

\section{Conclusion}

I must declare the authors' conceptualization of the mobile revolution excites me. Flashes of future research projects invaded my head as I read through the first chapter. So, I must admit that at first, I perceived the book very descriptive and information but did not expect to be presented with the complexities of the layers associated in the schema of the mobile multiplier effects of the mobile revolution. Despite my excitement, I was disappointed by the lack of acknowledgement of the sustainability implications that the growth of mobile device usage has on our environment. As we all know now, the majority of global citizens own mobile phones that are often quickly upgraded to the newest models, and disposing obsolete devices creates an excess of waste with environmental implications (Tan et al. 2017; however, MGDR has addressed this issue, see Kumar and Bhaskar 2016). Pivotal questions - that should have been addressed in this book - could have revolved around disposal, recycle, or repurposing strategies that need to be applied to manage the 42,000 tons of waste predicted by 2025 in countries such as China (Tan et al. 2017). China is key in addressing waste mobile phone (WMPs) due to it being the largest producer of mobile phones since 2004 as well as being the dumping ground for $70 \%$ of waste mobile phones (Yao et al. 2018). It is important to address mobile phone waste in this book due to the danger of highly toxic substances as well as opportunities to recycle retrievable elements such as silver, gold, and copper (Yin, Gao and Xu 2014). An 
additional element of rapid mobile technological growth, not discussed by the authors in this book, deals with the supplanting of individual regional cultures with technological innovative cultures with their own values and nuances (Husain and Kolesar 2018). A discussion on "what is lost" as a result of gaining the mobile device culture needs to be a part of a book such as this one.

In my opinion, in chapters two through five, the authors do a good job at presenting and explaining the complexities of mobile marketing strategies, mobile technology advances, and resulting privacy concerns. As a consumer, I was extremely eager to learn about all the innovative ways businesses are implementing mobile marketing strategies and underlying mechanisms that support successful mobile marketing strategies. I perceive that the authors - given the commercial nature of this book and its publisher - fail to go into the critical issue involved in businesses tracking consumer behavior via apps and other mobile technologies, under the auspices of meeting customer demands for information. As can be observed in the privacy issues which emerged during the 2016 presidential elections in the United States when Cambridge Analytica, a political consulting organization, impacted voter behavior by tapping into data mined from their Facebook social media platform (see Dholakia and Reyes 2018). A loss of consumer trust ensued from a wave of instant fear of being manipulated and an overall weakening of the financial markets (Peruzzi et al. 2018). With the rapid growth and development of mobile technology innovation, policies instituted to safeguard individuals' information will not only continue to play a central role in firms' success but also reflect national and global human values. 


\section{References}

Banerjee, Syagnik, Ruby Roy Dholakia and Nikhilesh Dholakia (2017), MPowering Marketing in a Mobile World. New York: Business Expert Press.

Belk, Russell (2017) "Collective Narcissism, Anti-Globalism, Brexit, Trump, and the Chinese Juggernaut," Markets, Globalization \& Development Review, 2 (3), Article 2. https://doi.org/10.23860/MGDR-2017-02-03-02

Dholakia, Nikhilesh and lan Reyes (2018), "Technology and Trump Triumph: A Social Theoretical Perspective", Working Paper, https://doi.org/10.13140/RG.2.2.15270.63048

Husain, Matt and Sebastian Kolesar (2018), "Can technology change the existing culture? Case study on Bangladesh's public sector," Development in Practice, 28 (1), 152-64. https://doi.org/10.1080/09614524.2018.1404555

Kumar, Bipul and Kalyan Bhaskar (2016), "Electronic Waste and Sustainability: Reflections on a Rising Global Challenge," Markets, Globalization \& Development Review, 1 (1), Article 5. https://doi.org/10.23860/MGDR-2016-01-01-05

Peruzzi, Antonio, Fabiana Zollo, Walter Quattrociocchi and Antonio Scala (2018), "How News May Affect Markets' Complex Structure: The Case of Cambridge Analytica," Entropy, 20 (10), 765. https://doi.org/10.3390/e20100765

Tan, Quanyin, Qingyin Dong, Lili Liu, Qingbin Song, Yangyang Liang and Jinhui Li (2017), "Potential recycling availability and capacity assessment on typical metals in waste mobile phones: A current research study in China," Journal of Cleaner Production, 148, 50917. https://doi.org/10.1016/j.jclepro.2017.02.036

Yao, Liming, Tingting Liu, Xudong Chen, Moudi Mahdi and Jingneng Ni (2018), "An integrated method of life-cycle assessment and system dynamics for waste mobile phone management and recycling in China," Journal of Cleaner Production, 187, 852-62. https://doi.org/10.1016/j.jclepro.2018.03.195

Yin, Jianfeng, Yingnan Gao, He Xu (2014), "Survey and analysis of consumers' behaviour of waste mobile phone recycling in China," Journal of Cleaner Production, 65, 517-25. https://doi.org/10.1016/j.jclepro.2013.10.006 